\title{
Optical properties of thin metal films
}

\author{
S.A. Kovalenko \\ Institute of Semiconductor Physics, NAS of Ukraine \\ 45, prospect Nauki, 252650 Kyiv, Ukraine
}

\begin{abstract}
Optical constants of metallic thin films made from: Ag, Au, Hf, Ir, Mo, Nb, Os, Pd, Pt, Re, $\mathrm{Rh}, \mathrm{Ru}, \mathrm{Ta}, \mathrm{W}, \mathrm{Zr}$ - were determined on the basis of measured index of refraction in region of wavelength $\lambda=24-1216 \AA$. Two types of relations were used for the calculation. Some of them were obtained, with taking into account that refractive index of absorbing medium can be presented in the form $\tilde{n}=n \pm i æ$. Other were obtained from Maxwell boundary condition.

Both approaches give rise to very close results for $æ$, however the dependences $n=f(\lambda)$ for $\lambda>200 \AA$ are essentially different. The reasons of such differences are discussed.
\end{abstract}

Keywords: thin layers, refraction coefficient, transmission coefficient, refraction index, absorption index

Paper received 02.09.99; revised manuscript received 29.09.99; accepted for publication 18.10.99.

\section{Introduction}

Investigations of optical properties of solids, including these of thin films, are aimed on: first, to obtain data for ascertaining their band structure; second, to determine such important parameters as refractivities and absorptivities necessary for designing devices and their elements.

Thin films of transparent and light absorbing materials are widely used in manufacturing interferential [1,2] and absorption [3,4] filters, in production of multi-layer mirrors $[5,6]$, polarizers $[7,8]$, light detectors $[9,10]$, in making antireflection coatings $[11,12]$. In all enumerated cases one needs to have exact data upon optical constants, $n$ and $æ$, of considered materials. For many years respective calculations were based on relationships that took into account repeated reflections and interference in a thin layer, and measured in experiment were intensities of light beams reflected and transmitted by the thin layer.

Formulae for intensities of a light wave reflected and transmitted by the layer include Fresnel coefficients of reflection and transmission for separate interfaces, namely: air (vacuum) - thin layer and thin layer - substrate.

In the case of absorbing media a refraction index is complex value $n+i æ$. Therefore, transition from transparent substance to absorbing one is usually postulated by the change $\tilde{n} \rightarrow n+i æ$, which cannot be considered as fully justified. It is this change that leads to meaningless results. For example, when a thickness of metal [13-15] and semiconductor [16-18] films tends to zero value, the refraction index increases to infinity, though it must approach to unity. Such monotonic thickness dependence of the refraction in- dex for thin absorbing films all the more cast doubt, because it must have at least one resonance maximum on the curve $n=f(d)$ that corresponds to a wedgeshaped layer thickness of which changes from zero to the value when this layer becomes absolutely transparent. Calculations fulfilled in accordance with above formulae [19] do not give such a maximum.

As it is known, investigations of metal optical properties are based on using ellipsometric method and measuring reflection coefficients for different angles of light incidence on mirror surface of a bulk sample. In these cases work formulae for $n$ and æ estimations are usually obtained by changing $\tilde{n} \rightarrow n+i$ æ. As a result, obtained values of $n$ in wide ranges of the optical spectrum are less than unity. As an example, we can give data $[20,21]$ for gold. Authors of the first paper considered the range of wavelength $0.5-1 \mu \mathrm{m}$ where $n$ monotonically decreases from 0.84 to 0.19 . In the second paper, measurements were performed in the range of $1.0-4 \mu \mathrm{m}$ that supplements the previous interval. $n$-values less than unity, were obtained in the region of $1.0-2.5 \mu \mathrm{m}$. With increasing wavelength, these raise from 0.224 to 0.82 . Thus, almost in the whole visible part of the spectrum, and in a considerable part of the infra-red region joining to it, the refraction index of gold is less than unit. We shall not give more numeric data by other authors for metals, semiconductors and dielectrics where values of $n<1$ are fixed in wide ranges of the optical spectrum. Moreover, in this paper we cite plots for $n=f(\lambda)$ dependences from [22] for 16 metals, and each of them has respective ranges of the spectrum.

The inequality $n<1$ is not alone to generate surprise. It 


\section{S.A. Kovalenko: Optical properties of thin metal films}

is difficult to imagine why it is intrinsic for wide spectral intervals while minimum $n$-values are usually met in the narrow range of frequencies corresponding to band halfwidths of electron or vibration transitions, that is, in the range of anomalous dispersion. As known, in this region, the group velocity with which light wave energy is transfered, is connected with refraction index dispersion through known relationship:

$$
\vartheta_{g r}=\frac{c}{n-\frac{d n}{d \lambda} \cdot \lambda}
$$

were $c-$ is the light velocity in vacuum. Since a phase velocity $\vartheta_{p h}=c / n$, then at frequencies where the sign of $d n / d \lambda$ is changed, i.e., $d n / d \lambda=0$,

$$
\vartheta_{g r}=\frac{c}{n}=\vartheta_{p h}>c
$$

taking into consideration that $n<1$. This result contradicts the special theory of relativity. By the way, if one takes for example the $n$-value of silver for the wavelength close to $0.5 \mu \mathrm{m}$ that is equal to 0.05 then the respective group velocity must exceed the light velocity in vacuum by 20 times! It is a fantastic result, there is nothing to say.

One example more [23] where authors cast doubt on legitimateness of using known Fresnel formulae. The authors had the task to measure depth of $X$-ray penetration into on oxide film on a silicon surface. Thicknesses of such films are small, so for increasing losses of an energy of an $X$ - ray beam at the cost of absorption and scattering, authors directed it at a grazing angle of incidence, and the depth of penetration was determined by the formula:

$$
\frac{1}{D}=2 \frac{\omega}{c} \operatorname{Im}\left(\sqrt{\varepsilon-\cos ^{2} \theta_{0}}\right) \text {. }
$$

Here, $\omega$ is an angular frequency, $c$ is the light velocity in vacuum, $\varepsilon$ is the dielectric permittivity of a $\mathrm{SiO}_{2}$ - layer, $\theta$ is an angle of grazing incidence. A large deviation of theoretically estimated and measured $D$-values is explained by authors as stemmed from non-Fresnel character of $\mathrm{Si}_{-} \mathrm{SiO}_{2}$ system behavior.

In our opinion, above examples are enough to motivate a necessity of searching new relationships which could fulfil the same functions as Fresnel formulae did for many years. The first steps along this line are made by T.O.Kudykina [25] who, using boundary conditions by Maxwell, obtained analogs to Fresnel formulae. Using these formulae and experimental data upon transmission and reflection by thin layers of atomic semiconductors obtained earlier [16-18], authors of [25] calculated thickness dependencies of optical constants $n$ and æ. As it was expected, each curve has a resonance maximum, and $n$ - values appear to tend to unity when $d$ goes to zero.

It would seem that such brilliant confirmation of the Kudykina formulae workability might interpose all on their places. However, as it will be seen below in discussing the problem, with these new opportunities some new questions arose.
In this paper, using reflection spectra of thin films for 16 metals optical data of which in the range of $\lambda=24$ $1216 \AA$ were obtained in [22], we calculated $n$ - and æ- values in accordance to old [19] and new [25] theories.

Investigated in [22] metal films were prepared by electron-beam sputtering in vacuum $\sim 5 \cdot 10^{-6}$ Torr on silicon substrates temperature of which was kept to be equal to $300{ }^{\circ} \mathrm{C}$. The thickness of the films was approximately $1000 \AA$.

\section{Work formulae}

Fig. 1 represents a scheme of a sample like those investigated in [22]. Let us consider that the substrate and air are transparent for wavelengths used in the work, i.e. $\mathfrak{x}_{1}=\mathfrak{x}_{3}=0$. It is also accounted that at thicknesses close to $1000 \AA$ the film-substrate boundary and, all the more, the substrateair one give negligable contribution into total-reflectivity. It is in such conditions this new theory is used for calculation $n$ and æ. Necessary relationships see below. These have the following appearance:

$$
\left.\begin{array}{c}
T_{14}=\frac{1-R_{34}}{1-R_{34} R R_{*}^{\prime}} T_{*}, \\
R_{14}=R_{*}+\frac{R_{34} T_{*}^{2}}{1-R_{34} R R_{*}^{\prime}},
\end{array}\right\}
$$

where $T_{14}, R_{14}$ are transmission and reflection coefficients of the system shown in Fig. 1 , respectively, $R_{34}=\left(\frac{n_{3}-1}{n_{3}+1}\right)^{2}$ is the Fresnel reflection coefficient of the substrate-air boundary;

$$
\begin{aligned}
& R_{*}=C / A ; \\
& R_{*}^{\prime}=B / A ; \\
& T_{*}=16 n_{3}\left(n_{2}^{2}+\mathfrak{x}_{2}^{2}\right) / A ; \\
& A=\rho \tau \exp \left(\gamma \mathfrak{x}_{2}\right)+\delta \sigma \exp (-\gamma \mathfrak{x})+2 s \cos n_{2} \gamma+2 t \sin n_{2} \gamma ; \\
& B=\sigma \rho \exp \left(\gamma \mathfrak{x}_{2}\right)+\delta \tau \exp \left(-\gamma \mathfrak{x}_{2}\right)+2 q \cos n_{2} \gamma-2 r \sin n_{2} \gamma ;
\end{aligned}
$$

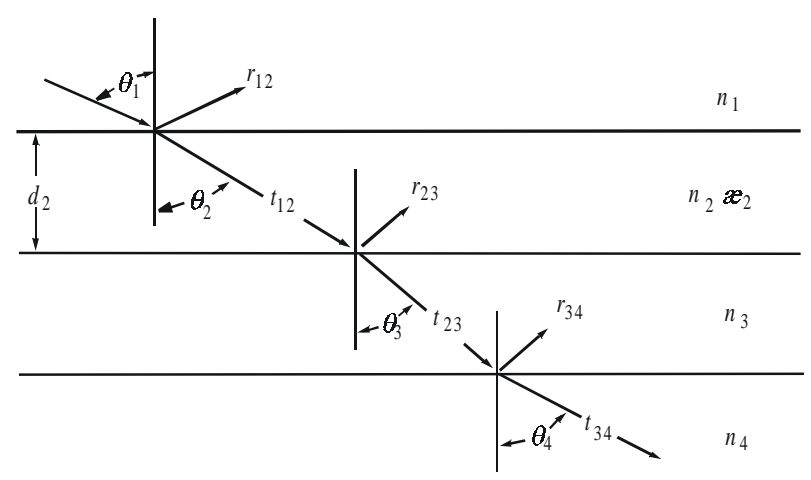

Fig.1. 


\section{S.A. Kovalenko: Optical properties of thin metal films}

$C=\delta \tau \exp (\gamma æ)+\sigma \rho \exp (-\gamma \mathfrak{x})+2 q \cos n_{2} \gamma+2 r \sin n_{2} \gamma$

$q=\left(n_{2}^{2}+\mathfrak{x}_{2}^{2}\right)\left(1+n_{3}^{2}\right)-\left(n_{2}^{2}+\mathfrak{x}_{2}^{2}\right)^{2}-n_{3}^{2}-4 n_{3} \mathfrak{x}_{2}^{2} ;$

$\rho=\left(n_{1}+1\right)^{2}+\mathfrak{x}_{2}^{2}$;

$s=\left(n_{2}^{2}+\mathfrak{x}_{2}^{2}\right)\left(1+n_{3}^{2}\right)-\left(n_{2}^{2}+\mathfrak{x}_{2}^{2}\right)^{2}-n_{3}^{2}+4 n_{3} \mathfrak{x}_{2}^{2} ;$

$\sigma=\left(n_{2}-n_{3}\right)^{2}+\mathfrak{x}_{2}^{2}$;

$r=2 \mathfrak{x}_{2}\left(n_{3}-1\right)^{2}\left(\mathfrak{x}_{2}^{2}+n_{2}^{2}+n_{3}\right) ;$

$\tau=\left(n_{2}+n_{3}\right)^{2}+\mathfrak{x}_{2}^{2}$;

$t=2 \mathfrak{x}_{2}\left(n_{3}+1\right)^{2}\left(\mathfrak{x}_{2}+n_{2}^{2}-n_{3}\right)$;

$\gamma=4 \pi d 2 / \lambda$

where $\lambda$ is the light wavelength in vacuum.

Both the traditional and the new theory start from the same relationships for reflection and transmission coefficients. These are as follows:

$$
\begin{aligned}
& R=\frac{r_{12}^{2}+r_{23}^{2} \exp (-2 \alpha d)-2 r_{12} r_{23} \exp (-\alpha d) \cos \psi}{1+r_{12}^{2} r_{23}^{2} \exp (-2 \alpha d)-2 r_{12} r_{23} \exp (-\alpha d) \cos \psi}, \\
& T=\frac{T_{12} T_{23} \exp (-\alpha d)}{1+r_{12}^{2} r_{23}^{2} \exp (-2 \alpha d)-2 r_{12} r_{23} \exp (-\alpha d) \cos \psi},
\end{aligned}
$$

where $\alpha=\frac{4 \pi æ_{2}}{\lambda}$,

$$
\psi=\frac{4 \pi}{\lambda} n_{2} d,
$$

Contrary to the old theory, in the new one, coefficients of reflection and transmission for separate boundaries airfilm and film-substrate do not include absorption indices at the normal angle of light beam incidence:

$$
\begin{aligned}
& r_{12}=\frac{n_{2}-n_{1}}{n_{2}+n_{1}} ; \quad r_{23}=\frac{n_{3}-n_{2}}{n_{3}+n_{2}}, \\
& t_{12}=\frac{2 n_{1}}{n_{2}+n_{1}} ; t_{23}=\frac{2 n_{1}}{n_{3}+n_{2}}, \\
& t_{21}=\frac{2 n_{2}}{n_{1}+n_{2}} ; \quad t_{32}=\frac{2 n_{3}}{n_{2}+n_{3}},
\end{aligned}
$$

It is worth to note that $R_{12}=\left(r_{12}\right)^{2}, R_{23}=\left(r_{23}\right)^{2}, T_{12}=t_{12} t_{21}$, $T_{23}=t_{23} t_{32}$.

Substituting $Z_{i}=\exp (-\alpha d)$, instead of Eqs (4) and (5), one can obtain quadratic equations relatively to $Z_{i}$ :

$$
\begin{aligned}
& R=\frac{r_{12}^{2}+r_{23}^{2} Z_{i}^{2}-2 r_{12} r_{23} \cos \psi \cdot Z_{i}}{1+r_{12} r_{23}^{2} Z_{i}^{2}-2 r_{12} r_{23} \cos \psi \cdot Z_{i}}, \\
& T=\frac{T_{12} T_{23} Z_{i}}{1+r_{12}^{2} r_{23}^{2} Z_{i}^{2}-2 r_{12} r_{23} Z_{i} \cos \psi} .
\end{aligned}
$$

Solving Eq. (12) yields roots for $Z$ :
$Z_{1,2}=b / 2 a \pm \sqrt{\frac{b^{2}}{4 a^{2}}-\frac{T}{a}}$,

where

$$
\begin{aligned}
& a=\operatorname{Tr}_{12}^{2} r_{23}^{2}, \\
& b=T_{12} T_{23}+2 T r_{12} r_{23} \cos \psi .
\end{aligned}
$$

Substituting $Z_{i}$ into Eq. (11), one can find $n_{2}$ - value as a root of the function

$F=R-\frac{r_{12}^{2}+r_{23}^{2} Z_{i}^{2}-2 r_{12} r_{23} \cos \psi \cdot Z_{i}}{1+r_{12} r_{23}^{2} Z_{i}^{2}-2 r_{12} r_{23} \cos \psi \cdot Z_{i}}$.

$Z_{i}-$ value must satisfy the condition $0<Z_{i}<1$. The sign before the root of the solution (13) is chosen in accordance with it.

After calculation of the $n_{2^{-}}$value we can find the $æ_{2^{-}}$ value using the next relation:

$\mathfrak{x}_{2}=\frac{-\lambda \cdot \ln Z_{i}}{4 \pi d}$,

which follows from (6) accounting the change $Z_{i}=\exp (-a d)$.

Spectral dependencies of $n$ and $æ$ calculated by using both the traditional formulae and those of the new theory are shown in Figs 2-17.

\section{Discussion}

The analysis of curves testifies that only for short wavelengths taken from the range of $\lambda=24-100 \AA$ refractive index values calculated using both theories practically coincide. At $\lambda>100 \AA$ these diverge, moreover, the new theory leads to considerably larger $n$ - values. For gold, silver, iridium, platinum and rhenium a maximum difference corresponds to wavelength taken from the interval $\lambda=530-550 \AA$. But for molibdenium, osmium, palladium, rhutenium and rhodium it is shifted up to $\lambda \approx 600 \AA$. The largest shift can be observed in cases of hafnium, tantalum $(\lambda \approx 1000 \AA)$, tungsten, zirconium and titanium $(\lambda \approx 1100-1200 \AA)$. It is most frequently at these wavelength that the new theory describes the $n$ - maximum which can be explained by the quantumsized resonance.

As for absolute values of refraction indices, the new theory leads only to $n>1$ in all range of wavelength used. At the same time, the old one leads to $n<1$ for all metals in wide ranges of the spectrum without any exception.

If we take into account that in the range of wavelengths $\lambda=24-1200 \AA$ quantum transitions realize between inner electron shells polarizabilities of which are considerably less than those of the outer ones, then $n$ - values exceeding unity can cast doubts.

A very interesting result was obtained for absorption indices. These almost coincide in all range of wavelength used, moreover, their absolute meanings do not cast doubts. Coincidence can be explained by respective estimations. If $\mathfrak{x}^{2} / n^{2}=0.05$ then there is no place for any divergence between old and new theories, but when $x^{2} / n^{2} \geq 0.25$ such difference will be considerable. 
S.A. Kovalenko: Optical properties of thin metal films
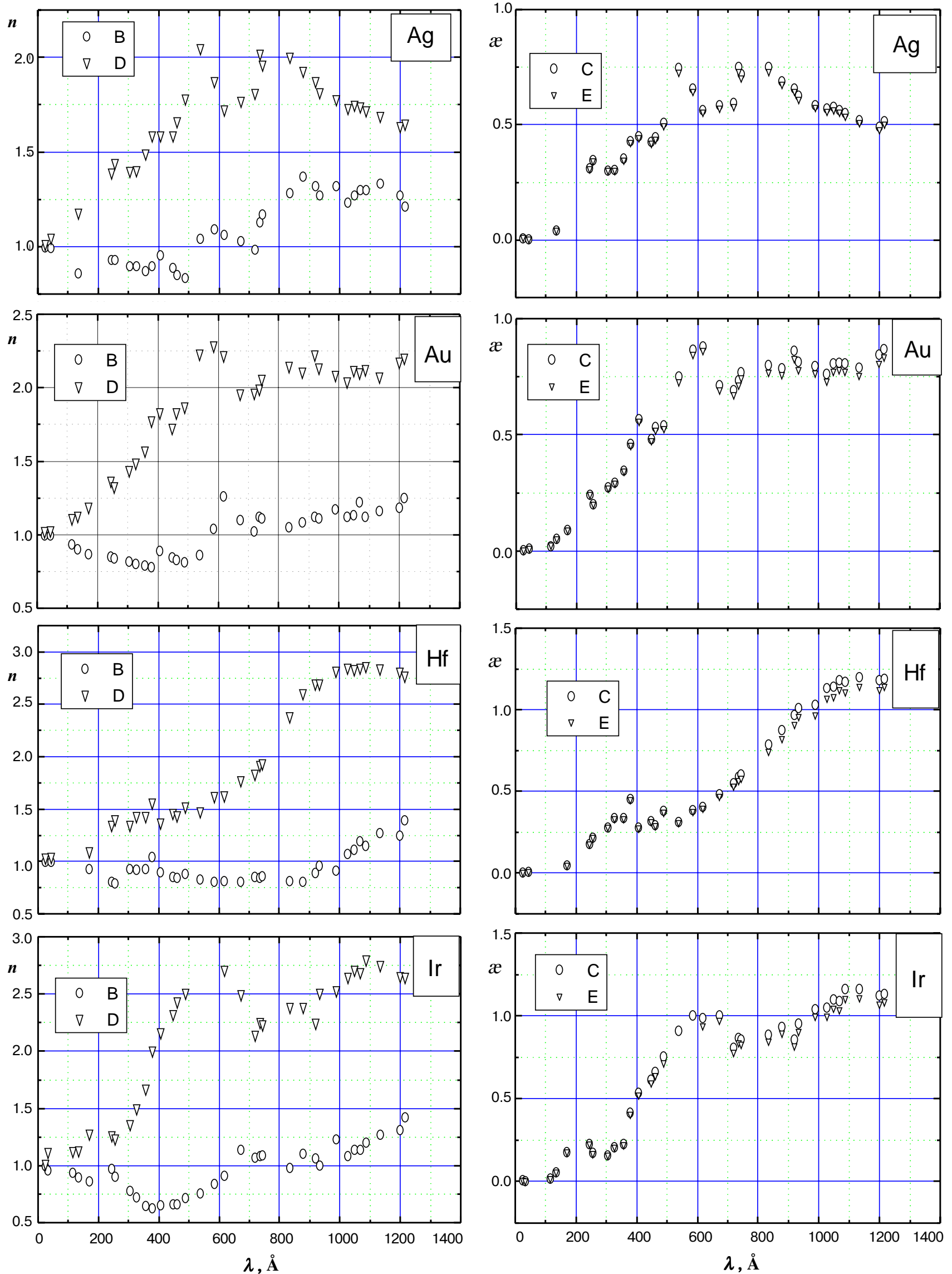

Figs 2-5. 
S.A. Kovalenko: Optical properties of thin metal films
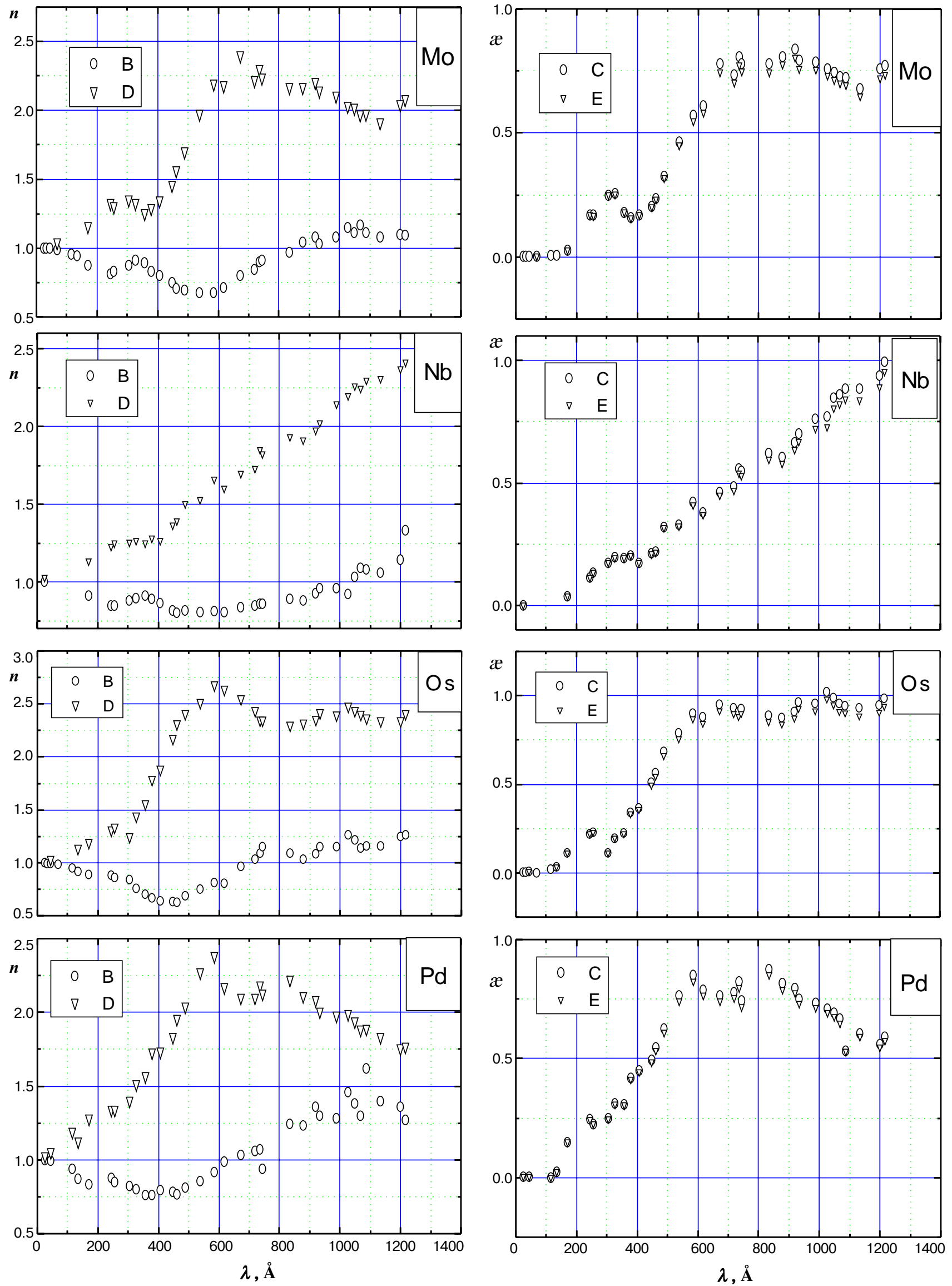

Figs 6-9. 
S.A. Kovalenko: Optical properties of thin metal films
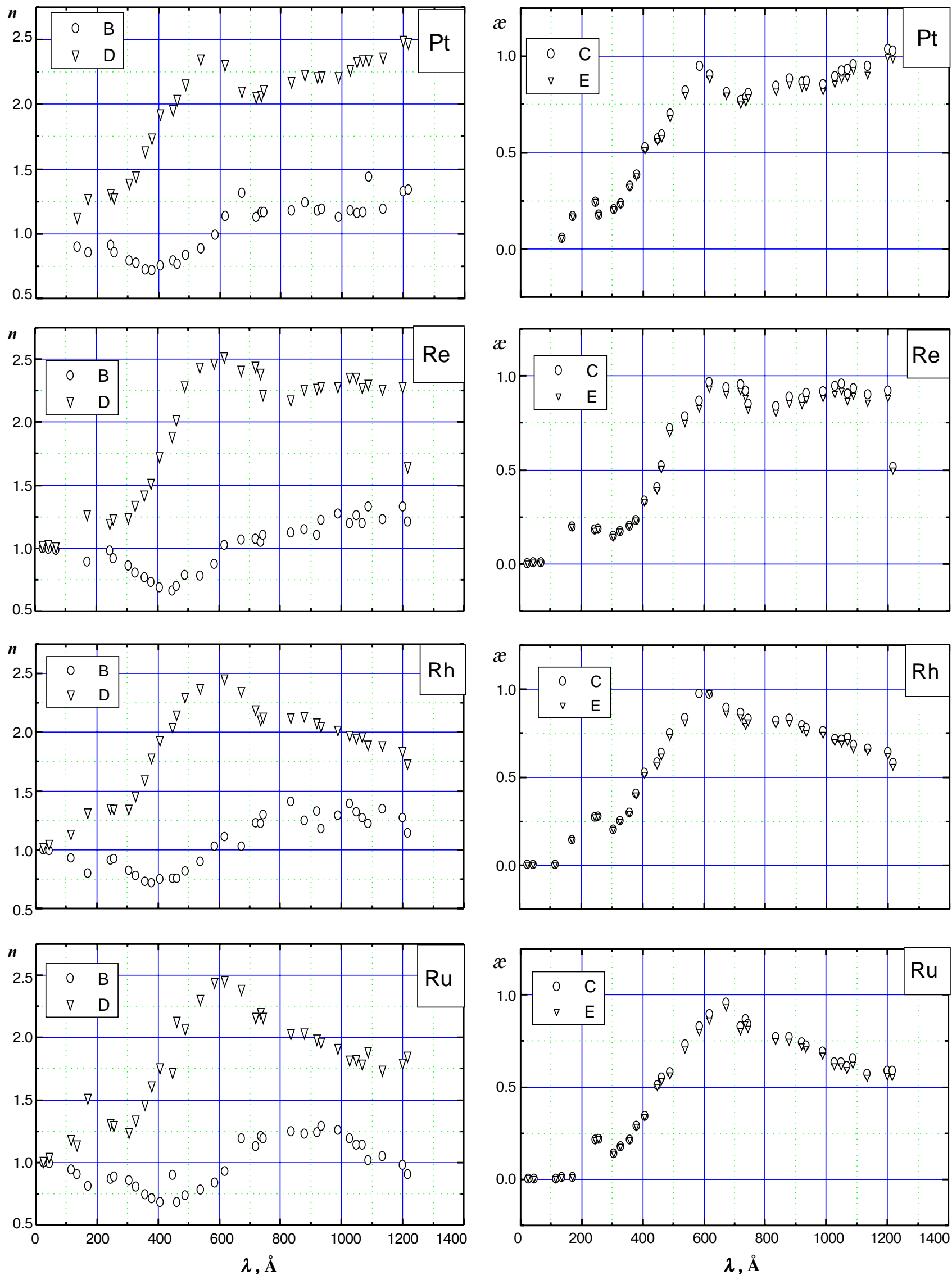

Figs 10-13. 


\section{S.A. Kovalenko: Optical properties of thin metal films}
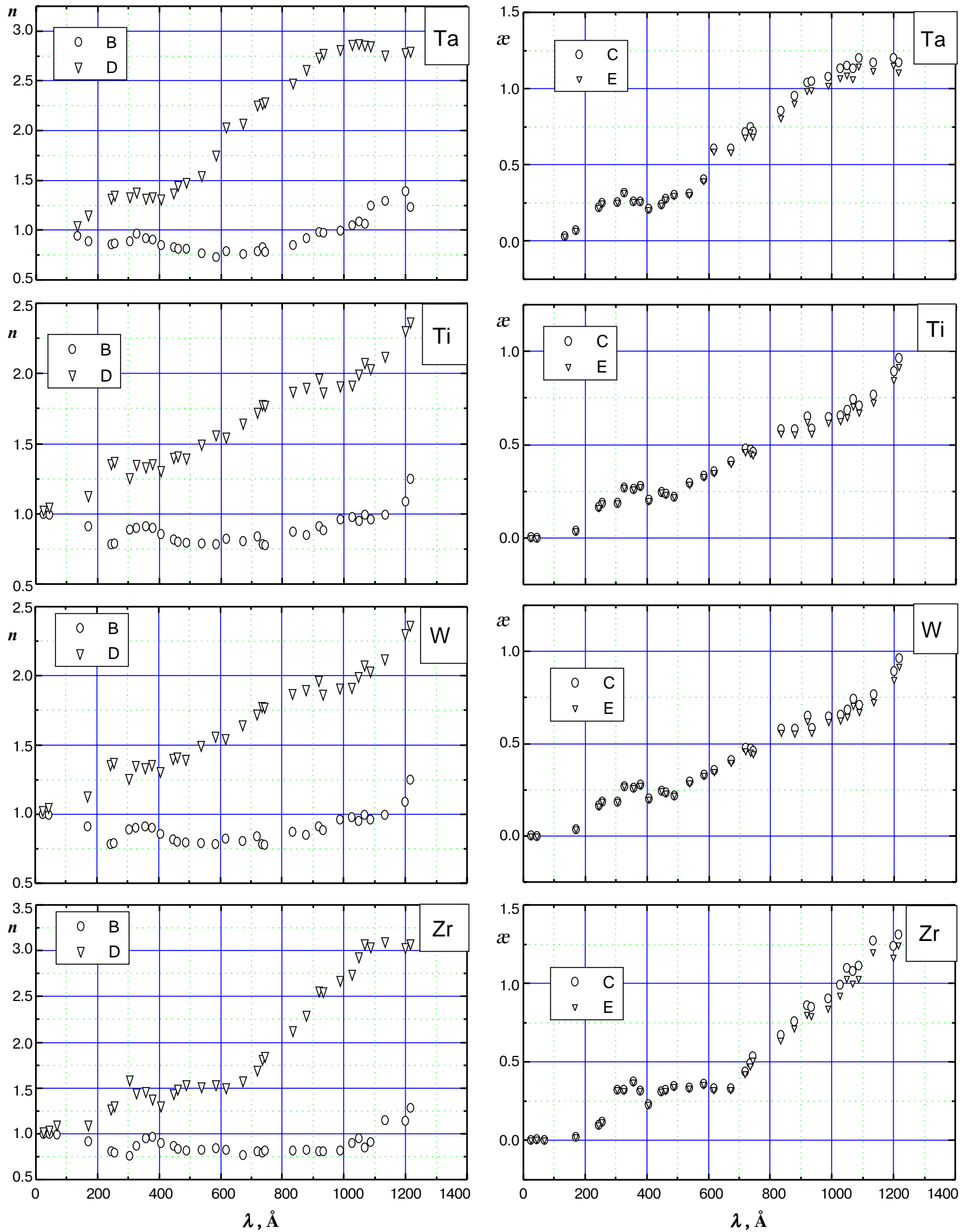

Figs 14-17. 


\section{S.A. Kovalenko: Optical properties of thin metal films}

\section{Conclusions}

1. Optical constants $n$ and $æ$ for 16 metals in the spectral region of $\lambda=24-1216 \AA$ are estimated. Experimental dependences of reflection coefficients on the wavelength are considered using two theories: the traditional one, where energetic coefficients of transmission and reflection on the boundary between two substances are calculated using substitutions $\tilde{n} \rightarrow n+i æ$ in the Fresnel formulae for transparent media, and the new theory in which the same coefficients are determined using formulae obtained from the Maxwell boundary conditions.

2. It is shown that coincidence of results takes place only for absorption indexes and for all metals in the whole spectral region. It is reasonable to believe that this coincidence has a random character and can be explained by a small æ-value in comparison with an $n$-value.

3. The accordance of $n=f(\lambda)$ curves with predictions of the new theory points on noticeable increase in $n$ - values with wavelength. However, an absolute $n$ - magnitude does not correlate with low polarizability of inner electron shells of atoms in the range of deep ultraviolet and $X$-rays. Therefore, the new theory predicts higher $n$-values.

4. Contrary to the previous conclusion, the traditional theory leads to lower $n$-values. There are wide spectral regions where $n<1$, i.e., one has the result deprived of real physical sense.

The author would like to emphasize his gratitude to Academician M.P.Lisitsa who is the supervisor and the author of the main idea of this work.

\section{References}

1. S.I. Gorban, S.V. Orlov, U.A. Pervak, I.V. Fekeshgasi. Multi ply kovering with broad zone reflection and transmition. // Ukrainskii fizicheskii zhurnal, 31, N 10, p.1494-96, (1986) (in Russian).

2. A.A. Aliseev, I.V. Ravadina. Prinsip of criation tunable interferation filter // Izvestiya vuzov. Ser. fizich., N 3, p.3, (1997) (in Russian)

3. L.I. Suslikov, V.P. Slivka, I.P. Lisitsa. Hard optical filter on the hirotropic crystals. Eiav, «Interpress LTD», 1998, p.292. (in Russian).

4. M. Wittmann, A. Panzkofer. Spectroscopic characterization of semiconductor doped colour filter glasses // Opt. Quant Electr., 27, N8, p.705, (1995)

5. I.P. Lisitsa, S.V. Irlov, U.A. Parvae, I.V. Fekeshgasi. Multi ply kovering with suppression of two ajasent zone of high reflection // Zhurnal prikladnoi spektroskopii, 47, N 2, p.283, (1987) (in Russian).
6. A.D. Petrenko, G.I. Orush, U.A. Parvae, I.V. Fekeshgasi. Reflection of ligh by kovering with fractal strukcher. // Optika i spektroskopiya, 83, N 3, p.416, (1997) (in Russian).

7. U.A. Parvae, I.V. Fekeshgasi. Spectral characteristics of prism interferention polarizer // Optika and spektroskopiya, 77, N 5, pp.843846, (1994) (in Russian).

8. U.A. Parvae, I.V. Fekeshgasi. Optimization of constructions of prism interferention polarizers and partishioners // Optika i spektroskopiya, 86, N 1, p.153, (1999) (in Russian).

9. W. Kruhler, Amorphous thin-film solar cells // Appl. Phys. A, 53, N 1, p.54, (1991)

10. F.I. Sebastian, N. Sivaramakishnan. The prospects of CdTe thin films as solar contour coatings // Thin Sol. Films, 202 , N 1, p.1, (1991)

11. R.E. Iuhamedov. Determination of optimalii index of refraction of phototropic kovering. //Opt-mah. fact., pub. 2, p.57, (1991) (in Russian)

12. A.N. Eitlikov, G. . Oarashchanei. Investigation of film optical constans, which use for synthesis of wide-band phototropic kovering. // Optika i spektroskopiya, 82, N 4, pp.653-659, (1997) (in Russian).

13. F. Goos, Zs. Phys., 100, N 1-2, 95, (1936); 105 , N 1, p.606, (1937)

14. I. Krautkramer, Proc. Phys. Soc., 32 , p.537, (1938)

15. B. Pogany, Ann. d. Phys., 49, p.537, (1916)

16. I.P. Lisitsa, N.G. Tsvelyh. Optics of thin film (II Te) // Optika and spektroskopiya, 4, N 3, pp.373-377, (1958) (in Russian).

17. I.P. Lisitsa, V.I. Iyevsky, N.G. Tsvelyh. Optics of thin film (III Se) // Optika i spektroskopiya, 5, N 2, pp.179-183, (1958) (in Russian).

18. I.P. Lisitsa, N.G. Tsvelyh. Optics of thin film (IV Ge) // Optika i spektroskopiya, 5, N 5, p.622, (1958) (in Russian).

19. H. Murman. Zeitschr. f. Phys. 80, s. 167, (1933)

20. Z. G. Sohulz and F. R. Tangherlini, JOSA, 44; N5, c.362, (1954)

21. G.O. Iitulevich, A.A. Shubin. Impact form of Fermy surfase of gold on the optical constant and Holl effect // Zhurnal eksperimental'noi i teoreticheskoi fiziki, 47, pub. 3(9), pp.840-847, (1964) (in Russian).

22. D.L. Windt, W.C. Cash, J.M. Scott, P. Arendt, B. Newnam, R.F. Fisher, A.B. Swartzlander, P.Z. Takacs, J.M. Pinneo. Optical constants for thin films of Ti, Zr, Nb, Mo, Ru, Rh, Pd, Ag, Hf, Ta, W, Re, Ir, Os, Pt, and $\mathrm{Au}$ from $24 \AA$ to $1216 \AA$ // Apl. Opt. 27, pp. 246-278, (1988)

23. A.I. Filatova, A.S. Shulakov, V.A. Lukyanov. Depth saping of reflection beam of smooth $\mathrm{x}$-ray radiation in mirror reflection term. // Solid State Phisycs, 7, pp.1360-1363, (1998) (in Russian).

24. T.A. Kudykina. Bondary conditions in case electromagnetic wave absorption // Phis. Stat. Sol (b) 160, pp. 365-373, 1990; Optical protertis of semiconductors (Ge, Se, GaAs, InSb) 165, pp. 591-598, (1991)

25. O.A. Eudyeina, I.P. Lisitsa. Dimention dependens of thin film Ge, Si, Se, Te // Optoelectronic and semiconductor technics, pp. 106-114, (1997) (in Russian). 